\title{
CLOSED FORM MODELS FOR PULL-IN VOLTAGE OF ELECTROSTATICALLY ACTUATED CANTILEVER BEAMS AND COMPARATIVE ANALYSIS OF CANTILEVERS AND MICROGRIPPER
}

\author{
Kalaiarasi Arcot Ramakrishnan - Hosimin Thilagar Srinivasan *
}

\begin{abstract}
Pull-in voltage Evaluation is significant for the design of electrostatically actuated MEMS devices. In this work simple closed form models are derived for computation of pull-in voltage of cantilever beams. These models are obtained based on five different capacitance models suitable for wide range of dimensions. Using these models pull-in voltages are computed for a range of dimensions and the results are compared with the experimentally verified $3 \mathrm{D}$ finite element analysis results. The results show that, for every given range of dimension, choice of the model changes for the evaluation of the pull-in voltage with a maximum deviation of $2 \%$. Therefore for a given range of dimension appropriate closed form model is to be chosen for accurate computation of pull-in voltage. Computation of pull-in voltage of microgripper further validates the closed form models. The results again show that for a given range of dimension only a particular model evaluates the pull-in voltage with less error.
\end{abstract}

K e y w or d s: capacitance models, cantilever beams, electrostatic actuators, FEM models, microgripper, MEMS, pull-in voltages

\section{INTRODUCTION}

Micro Electro Mechanical Systems (MEMS) capacitive type transducers are used to sense external mechanical excitation such as force, acceleration, as a change in capacitance. It requires electrical energy and this energy is applied as a constant voltage (or) constant charge [1].The voltage controlled parallel plate electrostatic actuator exhibits an important behavior called pull-in. Pullin voltage is one of the basic parameters of the design of many electrostatic MEMS devices. Accurate evaluation of the pull-in voltages is essential in the design of electrostatically actuated MEMS devices. In particular, in micromirrors, the designer avoids this instability in order to achieve stable motions. But in switching applications, the designer exploits this effect to optimize the performance of the device [3]. The pull-in problem of beams cannot be solved analytically and numerical techniques using Finite Element Analysis (FEA) are computationally expensive as they are time consuming. Closed form expressions are very useful for designers as they provide some basic information regarding pull-in voltage. Many attempts have been made by several authors [10-19] to derive a closed form expression for the pull-in voltage. Chowdhry et al [10] has derived closed form model for pull-in voltage calculation by considering Meji's and Fokkema's capacitance formula [5] as better capacitance model [2]. Here the investigations were done for selective dimensions of cantilever beam. But further investigation for wide range of dimension of cantilever beam shows that the closed form model used in paper [10] alone is not sufficient. Different capacitance models are available in literature [49] Chang's model [4] is very accurate [2]. But Chang's model is computationally expensive. Therefore this paper takes into consideration of all other capacitance models available in literature [5-9]. Based on these models pull-in voltage of cantilever beam is computed for wide range of dimensions. A detailed comparative analysis is done, by comparing the pull-in voltages obtained from the closed form models with CoventorWare FEA model results. The results show that, for a given range of dimension one particular model suits better for the evaluation of the pull-in voltage of cantilever beam with a maximum deviation of $2 \%$ as compared with the experimentally verified FEA results. Further the model's validity is verified with comparing the pull-in voltage calculated for an electrostatically actuated microgripper, [20].

Chowdhry et al [10] has derived closed form model for pull-in voltage calculation by considering Meji's and Fokkema's capacitance formula [5] as better capacitance model [2]. Here the investigations were done for selective dimensions of cantilever beam. But further investigation for wide range of dimension of cantilever beam shows that the closed form model used in paper [10] alone is not sufficient. Different capacitance models are available in literature [4-9] among which Chang's model [4] is very accurate [2]. But chang's model is computationally expensive. Therefore this paper takes into consideration of all other capacitance models available in literature [59]. Based on these models pull-in voltage of cantilever beam is computed for wide range of dimensions. Moreover the suitability of each model for the calculation of pull-in voltage has also been investigated in the present paper. A detailed comparative analysis is done, by comparing the pull-in voltages obtained from the closed form

* Department of Electrical and Electronics Engineering, College of Engineering, Anna University, Chennai 600025, India, kalai06@gmail.com, shthilagar@gmail.com 


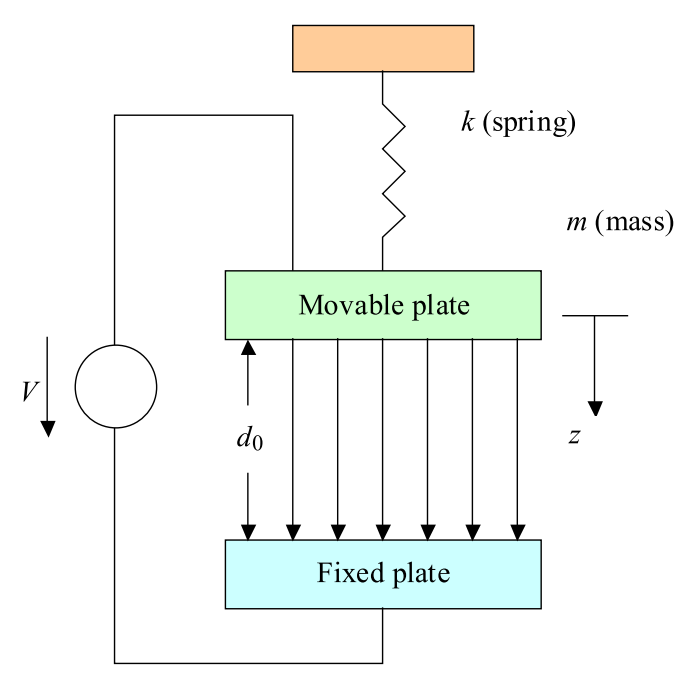

Fig. 1. The basic electrostatic actuator - Lumped parameter model of parallel plate electrostatic actuator

models with CoventorWare ${ }^{\mathrm{TM}}$ FEA model results. The results show that, for a given range of dimension one particular model suits better for the evaluation of the pull-in voltage of cantilever beam with a maximum deviation of $\pm 2 \%$ as compared with the experimentally verified FEA results.

\section{CANTILEVER BEAM PULL-IN VOLTAGE MODEL}

The lumped parameter model of the actuator is shown in Figure 1. It is also assumed that the movable plate's elastic restoring force (spring force) is linear. By neglecting any damping within the system, the equation of motion of the movable plate due to an electrostatic force $f_{E}$ can be expressed as,

$$
m \frac{\mathrm{d}^{2} z}{\mathrm{~d} t^{2}}+k z=f_{E}
$$

where $m$ - is the mass and $k$ - is the spring constant. The electrostatic attraction force $\left(f_{E}\right)$ of the plate can be found by differentiating the stored energy of the capacitor with respect to the position of the movable plate and is given as

$$
\begin{gathered}
f_{E}=-\frac{\mathrm{d}\left(\frac{1}{2} C V^{2}\right)}{\mathrm{d} z}=\frac{\varepsilon_{0} A V^{2}}{2\left(d_{0}-z\right)^{2}} \\
C=\frac{\varepsilon_{0} A}{d_{0}-z}
\end{gathered}
$$

where $C$ - is the capacitance, $\varepsilon_{0}$ - is the permittivity of free space, $A$ - is the beam area, $d_{0}-$ is the distance between movable and fixed plates and $z$ - is the displacement of the movable plate; and the spring force (elastic restoring force) is represented as

$$
f_{M}=k z
$$

where $f_{M}$ - is the mechanical elastic restoring force. At equilibrium $f_{\text {electrostatic }}=f_{\text {spring }}$. At a static equilibrium $f_{M}=f_{E}$.

If the electrostatic force is increased by increasing the applied voltage and if that force is greater than the elastic restoring force, the equilibrium is lost and the movable plate will collapse on the fixed ground plate. This phenomenon is known as pull-in. Hence at equilibrium

$$
k z=\frac{\varepsilon_{0} A V^{2}}{2\left(d_{0}-z\right)^{2}},
$$

To evaluate the pull-in voltage $\left(V_{P I}\right)$ the above equation is solved as in [1], giving

$$
V_{P I}=\sqrt{\frac{8 k d_{0}^{3}}{27 \varepsilon_{0} A}}
$$

from where the spring constant of the movable plate is found easily

$$
k=\frac{27 \varepsilon_{0} A V_{P I}^{2}}{8 d_{0}^{3}} .
$$

If the applied voltage is increased beyond the pull-in voltage, the resulting electrostatic force will overcome the elastic restoring force and will cause the movable plate collapse on the fixed ground plane and the capacitor will be short circuited. By expanding (3) using a Taylor series approximation about a distance $z_{0}$ as outlined in paper [12], equation the following can be derived

$$
\begin{aligned}
f_{E}=\frac{\varepsilon_{0} A V^{2}}{2\left(d_{0}-z\right)^{2}}=\left.\frac{\varepsilon_{0} A V^{2}}{2\left(d_{0}-z\right)^{2}}\right|_{z=z_{0}} \\
\quad+\left.\frac{\varepsilon_{0} A V^{2}(-2)(-1)}{2\left(d_{0}-z\right)^{3}}\right|_{z=z_{0}}\left(z-z_{0}\right)+\ldots,
\end{aligned}
$$

After simplification and rearrangement of the terms in (8) we find

$$
f_{E}=\frac{\varepsilon_{0} A V^{2}}{2\left(d_{0}-z\right)^{2}}\left[1+2 \frac{z-z_{0}}{d_{0}-z_{0}}+\ldots\right]
$$

By substituting $F_{E}$ from (9) into (1), we have

$$
m \frac{\mathrm{d} d^{2}}{\mathrm{~d} t^{2}}+k z=\frac{\varepsilon_{0} A V^{2}}{2\left(d_{0}-z_{0}\right)^{2}}\left[1+2 \frac{z-z_{0}}{d_{0}-z_{0}}+\ldots\right] .
$$

and after rearrangement

$$
\begin{aligned}
m \frac{\mathrm{d}^{2} z}{\mathrm{~d} t^{2}}+(k- & \left.\frac{\varepsilon_{0} A V^{2}}{2\left(d_{0}-z_{0}\right)^{3}}\right) z \\
& =\frac{\varepsilon_{0} A V^{2}}{2\left(d_{0}-z_{0}\right)^{2}}\left[1-2 \frac{z_{0}}{d_{0}-z_{0}}+\ldots\right] .
\end{aligned}
$$

From (11) it is evident that the electrostatic attraction force effectively modifies the spring constant of the movable plate and the term within the parenthesis on the 

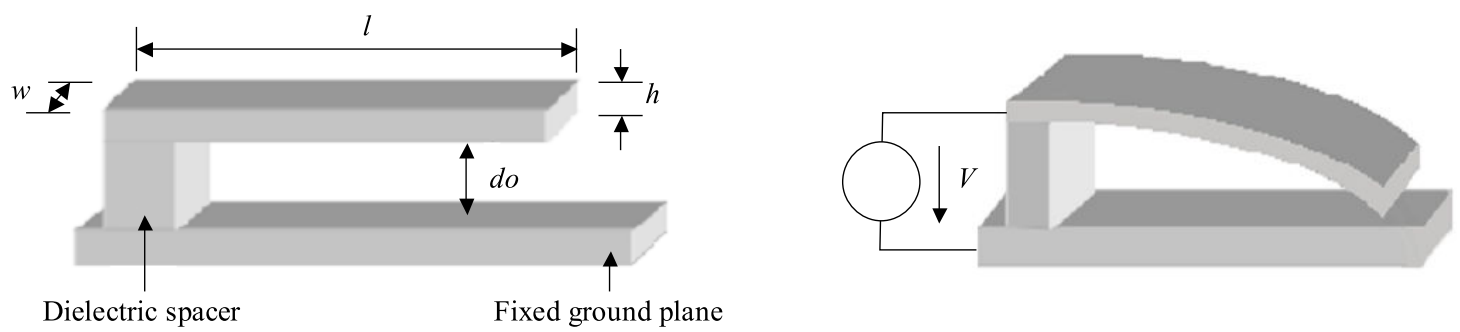

Fig. 2. (a) - a cantilever beam separated from a fixed ground plane by a dielectric spacer, (b)- deformation of the beam due to electrostatic force

\section{CANTILEVER BEAM}

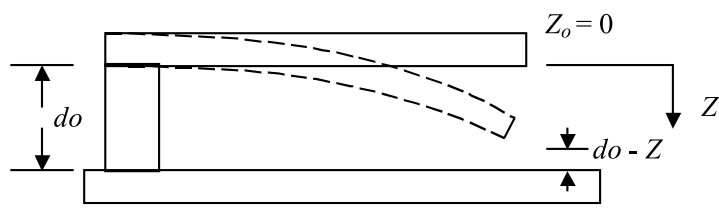

Fig. 3. Linearization of the electrostatic force about the zero deflection point $\left(z_{0}=0\right)$

left-hand side of (11) represents the effective spring constant at a specific voltage. The amount of modification is termed as the spring softening and can be expressed as

$$
k_{\mathrm{soft}}=\frac{\varepsilon_{0} A V^{2}}{2\left(d_{0}-z\right)^{3}} .
$$

For a cantilever beam as shown in, Fig. 2(a), the electrostatic force becomes increasingly non-uniform as the beam deforms as shown in Fig. 2(b). As a result, the tip of the cantilever will experience a higher attractive force comparing to the region closer to the fixed end. Following [11], an expression for a uniform pressure causing a cantilever tip deflection of $\mathrm{z}$ can be derived as

$$
P=\frac{k z}{w l}=\frac{2}{3} \frac{\tilde{E} h^{3}}{l^{4}} z
$$

where $\tilde{E}$ is the plate modulus $E /\left(1-\nu^{2}\right)$, and $E-$ is the Young's Modulus, $\nu$ - is the Poisson's Ratio, $w-$ is the width of beam, $l$ - is the beam length, and $h$ is the height of the beam.

A uniform linearized model of the electrostatic force can be obtained from (11) and (12) by linearizing the electrostatic force about zero deflection point $\left(z_{0}=0\right)$ as shown in Fig. 3. Since before any deflection the beam surface is assumed to be planar, the parallel-plate approximation can easily be applied without causing any significant error if air-gap thickness $\left(d_{0}\right)$ is very small compared to the lateral dimensions of the beam. Linearizing (11) about the point $z_{0}=0$, the following equation is arrived

$$
m \frac{\mathrm{d}^{2} z}{\mathrm{~d} t^{2}}+\left(k-k_{\mathrm{soft}}\right) z=\frac{\varepsilon_{0} w l V^{2}}{2 d_{0}^{2}}
$$

with the appropriate spring softening

$$
k_{\mathrm{soft}}=\frac{\varepsilon_{0} w l V^{2}}{2 d_{0}^{3}} .
$$

Rearranging (14) and neglecting the time-dependent term in a static case, the force equilibrium relation for any displacement $z$ can be obtained. It is given by

$$
k z=\frac{\varepsilon_{0} w l V^{2}}{2 d_{0}^{2}}+k_{\mathrm{soft}} z .
$$

The effective linearized uniform electrostatic pressure on the beam can be found from (16) and it is given as

$$
P_{\text {eff }}=\frac{F_{E-\text { linear-uniform }}}{w l}=\frac{\varepsilon_{0}}{V^{2}} 2 d_{0}^{2}+\frac{k_{\text {soft }} z}{w l} .
$$

Substituting $k_{\text {soft }}$ from (15) into (17) and replacing $z$ in (17) by the pull-in deflection $z=1 / 3 d_{0}$, the pull-in electrostatic pressure $P_{\mathrm{PI} \text {-electrostatic }}$ can be evaluated as

$$
P_{P I-\text { electrostatic }}=\frac{5 \varepsilon_{0} V_{P I}^{2}}{6 d_{0}^{2}}
$$

where $V_{P I}$ represents the pull-in voltage. In order to compensate for the error that arises due to neglecting higher order terms in Taylor series expansion and error due to the linearization, a compensation factor $\left(c_{f}\right)$ has been determined by a trial and error method, while comparing the results with CoventorWare ${ }^{\mathrm{TM}}$ FEA model results. The compensation factor is applied to (18) to get

$$
P_{P I-\text { electrostatic }}=c_{f} \varepsilon_{0} V_{P I}^{2} \frac{5}{6 d_{0}^{2}} .
$$

By substituting the pull-in deflection, $z=1 / 3 d_{0}$, in (13), the elastic restoring pressure at pull-in is obtained.

$$
P_{P I-\text { elastic }}=\frac{2}{3} \frac{\tilde{E} h^{3}}{l^{4}} \frac{d_{0}}{3}=\frac{2 \tilde{E} h^{3} d_{0}}{9 l^{4}} .
$$

Since at pull-in equilibrium, the electrostatic pressure is just counterbalanced by the elastic restoring pressure $\left(P_{P I-\text { electrostatic }}=P_{P I \text {-elastic }}\right)$, equations $(19)$ and $(20)$ can now be solved simultaneously to yield the final closedform expression for the pull-in voltage as

$$
V_{P I}=\frac{h}{l^{2}} \sqrt{\frac{0.222 h}{c_{f}(5 / 6) d_{0}} \frac{\tilde{E}}{\varepsilon_{0}}} .
$$




\section{CLOSED FORM MODELS OF PULL-IN VOLTAGE FROM CAPACITANCE MODELS}

\subsection{Capacitance Models}

This section presents the closed form models of pullin voltage obtained from various models of capacitances available in literature [5-9], using the procedure explained in the above section. These capacitance models are basically parallel plate models with fringing fields. These capacitance models are found to be suitable for wide range of dimensions of parallel plate capacitors [2]. Based on these capacitance models the closed form models of pullin voltage are derived and presented in Section 3.2. Further its suitability for different range of dimensions is extensively investigated in Section 4.

\subsubsection{Mejis and Fokkemas model (Model 1)}

Meji and Fokkema [5] improved Sakurais model [8] by extending the empirical expression.

$$
c=\varepsilon_{0} l\left[\frac{w}{d_{0}}+0.77+1.06\left(\frac{w}{d_{0}}\right)^{0.25}+1.06\left(\frac{h}{d_{0}}\right)^{0.25}\right] .
$$

The first term describes the parallel-plate capacitor and the other allows for all side effects.

\subsubsection{Yuan and Tricks model (Model 2)}

Yuan and Trick [6] presented simple analytic approximation. They replaced the rectangular line profile with an "oval" one. The resulting capacitance is given by

$c=$

$$
\varepsilon_{0} l\left[\frac{w-h / 2}{d_{0}}+\frac{2 \pi}{\log \left(1+\frac{2 d_{0}}{h}+2 \sqrt{\frac{d_{0}}{h}\left(\frac{d_{0}}{h}+1\right)}\right)}\right] .
$$

With reference to $[2,6]$, a maximum error of 10 percent with respect to Changs formula [4] is stated.

\subsubsection{Elmasrys model (Model 3)}

$$
c=\varepsilon_{0} l\left[\frac{w}{d_{0}}+2 \log \left(1+\frac{h}{d_{0}}\right)+\frac{2 h}{d_{0}} \log \left(1+\frac{w / 2}{h+d_{0}}\right)\right] .
$$

The first term of (24) represents the parallel plate capacitance, the second represents the capacitance associated with the side walls, and the third term represents the capacitance associated with the top side of the beam.

\subsubsection{Sakurai and Tamarus model (Model 4)}

$$
c=\frac{\varepsilon_{0} l}{d_{0}}\left[1.15 \frac{w}{d_{0}}+2.80\left(\frac{h}{d_{0}}\right)^{0.222}\right] .
$$

The first term of (25) represents the capacitance of the top and side walls of the beam and the second term represents the side wall contribution.
3.1.5 Palmers model (Model 5)

$$
c=\frac{\varepsilon_{0} l w}{d_{0}}\left[1+2 \frac{d_{0}}{\pi w}+2 \frac{d_{0}}{\pi w} \log \frac{\pi w}{d_{0}}\right] .
$$

This model includes the parallel plate capacitance and includes the fringing field capacitance due to the width of the capacitor. But it neglects the capacitance due to the lateral surfaces.

\subsection{Pull-in Voltage}

Based on the above capacitance models the closed form models of the pull-in voltages are derived based on the procedure outlined in [12], whose final form is presented in Appendix. It is to be noted that here shown model 1 has been already discussed in [10]. Now, as explained before a compensation factor is applied for every model whose value is the same irrespective of the change in dimensions.

\subsection{FEA Based Computation of Pull-in Voltage}

The cantilever beam is modeled and analyzed for wide range of dimensions using Finite Element Analysis (FEA) based software platform CoventorWare. Meshing is done based on mesh convergence study. Cosolve (coupled analysis of MemMech and MemElectro) is one of the solvers of CoventorWare that is used to detect the pull-in voltage. The FEA model used in this study is shown Fig. 4.

\section{MODEL VALIDATION}

Pull-in voltages of cantilever beam have been computed using the models in Section 3 over wide range of dimensions. The specific ranges used for the calculation of pull-in voltage are based on the paper [2].

Here the values of $d_{0}$ are selected as very low to match the parallel plate approximation as considered in the derivation of closed form models. The pull-in voltage for different ranges are computed and presented in Tab. 1 trough Tab.3.

The values of pull-in voltage are validated using Cosolve FEA results; where in Cosolve FEA results have been already verified with the experimental results $[6,10]$. It was reported that the difference of the experimentally measured values and Cosolve FEA results as $0.83 \%$. Therefore the authors of [10] have used Cosolve FEA results as a bench mark. Though it is claimed that the accuracy of pull-in voltage obtained from FEA based models are best compared with closed form models $[2,10]$, the former is time consuming compared to the later.

Here for each closed form model the compensation factor applied is unique across all dimensions and it has taken care of the reduction of error substantially. If the closed form model's error within $2 \%$ is considered as less error [10], then that model can be considered as a better 
Table 1. Pull-in voltage and \%-error comparison for $w / d_{0} \leq 2$

\begin{tabular}{ccccc}
\hline \multicolumn{5}{c}{ Common parameters } \\
$h=1.3 \mu \mathrm{m}, d_{0}=0.75 \mu \mathrm{m}$, Poisson's ratio $v=0.06$, \\
Young's modulus $E=169 \mathrm{GPa}, l=100 \mu \mathrm{m}$. \\
\hline$w / d_{0}$ & 0.667 & 1.2 & 1.333 & 2 \\
\hline$\underline{V_{P I 1}}$ & 5.2554 & 6.0076 & 6.1286 & 6.5473 \\
$\underline{\text { \%-error }}$ & $\underline{0.4856}$ & $\underline{1.4185}$ & $\underline{0.6871}$ & $\underline{0.1937}$ \\
$V_{P I, 2}$ & 5.1518 & 5.9663 & 6.0977 & 6.5512 \\
\%-error & 1.4955 & 2.0957 & 1.1872 & 0.1339 \\
$V_{P I, 3}$ & 5.1956 & 5.8984 & 6.0132 & 6.4249 \\
\%-error & 0.6568 & 3.2098 & 2.5568 & 2.0597 \\
$V_{P I, 4}$ & 5.3769 & 5.9885 & 6.0806 & 6.3840 \\
\%-error & 2.808 & 1.7308 & 1.4648 & 2.6774 \\
$V_{P I, 5}$ & 5.4816 & 6.1 & 6.1930 & 6.4996 \\
\%-error & 4.8103 & 0.0985 & 0.3559 & 0.9206 \\
Cosolve FEA & 5.23 & 6.094 & 6.171 & 6.56 \\
\hline
\end{tabular}

Table 2. Pull-in voltage and \%-error comparison for $w / d_{0} \geq 10$

\begin{tabular}{cccccccc}
\hline Common: $h=1.3 \mu \mathrm{m}, d_{0}=0.75 \mu \mathrm{m}, l=100 \mu \mathrm{m}$, \\
Poisson's ratio $v=0.06$, \\
Young's modulus $E=169 \mathrm{GPa}$, \\
\hline$w / d_{0}$ & 10 & 15 & 28 & 30 & 40 & 50 \\
\hline$V_{P I, 1}$ & 7.52 & 7.61 & 7.74 & 7.74 & 7.77 & 7.80 \\
\%-error & 8.33 & 9.66 & 10.02 & 9.66 & 10.12 & 10.41 \\
$V_{P I, 2}$ & 7.56 & 7.67 & 7.77 & 7.78 & 7.81 & 7.82 \\
\%-error & 9.01 & 10.28 & 10.51 & 10.13 & 10.53 & 10.77 \\
$V_{P I, 3}$ & 7.79 & 8.09 & 8.52 & 8.56 & 8.73 & 8.86 \\
\%-error & 13.39 & 17.54 & 21.15 & 22.46 & 24.91 & 26.66 \\
$\underline{V_{P I, 4}}$ & 6.70 & 7.06 & 7.11 & 7.11 & 7.13 & 7.14 \\
$\underline{\text { \%-error }}$ & $\underline{0.83}$ & $\underline{1.44}$ & $\underline{1.10}$ & $\underline{0.71}$ & $\underline{0.92}$ & $\underline{1.05}$ \\
$\underline{V_{P I, 5}}$ & 7.11 & 7.17 & 7.23 & 7.23 & 7.25 & 7.26 \\
\%-error & 2.55 & 3.16 & 2.80 & 2.40 & 2.62 & 2.75 \\
Cosolve & 6.94 & 6.95 & 7.03 & 7.06 & 7.06 & 7.06 \\
FEA & & & & & \\
\hline
\end{tabular}

model. The results of the better suited model amongst them are shown underlined in Tab.1 through Tab 3.

Table 1 shows the Pull-in voltage and its \%-error for $w / d_{0} \leq 2$. In this range $V_{P I, 1}$ s error is within $2 \%$. Therefore for the range of $w / d_{0} \leq 2, V_{P I, 1}$ is a better suited model.

Table 2 shows the pull-in voltage and the \%-error for the range of $w / d_{0} \geq 10$. In this range $V_{P I, 4} \mathrm{~S}$ error is less compared to other models. Therefore in the range of $w / d-0 \geq 10$, it is concluded that $V_{P I, 4}$ is a better suited model.

Table 3 shows that $V_{P I, 1}$ percentage error is less compared with other models for the range of $w \geq h / 2$ and $h \approx d_{0}$. If the thickness of the beam is equal to the gap between the substrate and the beam, and $w \geq h / 2$, the model $V_{P I, 1}$ is a better suited model.
Table 3. Pull-in voltage and \%-error comparison for $w \geq h / 2$

$$
\text { Common parameters }
$$

$h=0.75 \mu \mathrm{m}, d_{0}=0.75 \mu \mathrm{m}$, Poisson's ratio $v=0.06$, Young's modulus $E=169 \mathrm{GPa}, l=100 \mu \mathrm{m} .\left(h \approx d_{0}\right)$

\begin{tabular}{ccccc}
\hline$w$ & 0.375 & 1.5 & 2.5 & 3.5 \\
\hline$\underline{V_{P I, 1}}$ & 2.26 & 2.95 & 3.11 & 3.19 \\
$\frac{\text { \%-error }}{V_{P I, 2}}$ & $\underline{0.23}$ & $\underline{0.73}$ & $\underline{0.57}$ & $\underline{1.99}$ \\
\%-error & 5.56 & 1.75 & 0.81 & 3.19 \\
$V_{P I, 3}$ & 2.26 & 2.97 & 3.18 & 3.31 \\
\%-error & 0.08 & 0.14 & 1.78 & 5.83 \\
$V_{P I, 4}$ & 2.27 & 2.83 & 2.94 & 2.99 \\
\%-error & 0.14 & 4.63 & 5.83 & 4.17 \\
$V_{P I, 5}$ & 2.25 & 2.85 & 2.97 & 3.03 \\
\%-error & 0.89 & 4.06 & 4.92 & 3.06 \\
Cosolve FEA & 2.27 & 2.97 & 3.13 & 3.13 \\
\hline
\end{tabular}

Table 4. Pull-in voltage and \%-error comparison for microgripper

Finger: length $15 \mu \mathrm{m}$, width $1 \mu \mathrm{m}$, height $1.2 \mu \mathrm{m}$,

Extension arm length $101 \mu \mathrm{m}$,

Beam width $1.2 \mu \mathrm{m}, w / d_{0}=0.1$

\begin{tabular}{cccccc}
\hline Models & $V_{P I, 1}$ & $V_{P I, 3}$ & $V_{P I, 4}$ & $V_{P I, 5}$ & Cosolve \\
Pull-in & 29.33 & 38.90 & 24.61 & 27.35 & 29.69 \\
Voltage & & & & & \\
Error \% & 1.20 & 7.89 & 31.03 & 17.09 & - \\
\hline
\end{tabular}

Moreover, Fig. 5 shows the pull-in voltage versus width of the beam with respect to both the closed form model results and the CoventorWare results. Here the results are plotted for further a wide range; width varying from $0.25 \mu \mathrm{m}$ to $50 \mu \mathrm{m}$. It could be seen that the models 1 , 4 and 5 are found to be closer to CoventorWare results. This corroborates with the results already presented in Tab. 1 through Tab. 3. Therefore it could be concluded that a particular model is better suited for a particular dimension but indeed not in the entire range. Paper [10] presented a closed form model for the computation of pull-in voltage based on Meji's and Fokkema's capacitance model. Investigation shows that this model is suitable only for particular dimensions but not for wide range of variation in dimension of cantilever beam. Therefore appropriate model has to be chosen for the appropriate dimension of cantilever beam for the calculation of pull-in voltage.

\section{MICROGRIPPER PULL-IN VOLTAGE ANALYSIS}

The proposed closed form models were also applied to find the pull-in voltage of electrostatically actuated microgripper. Figure 6 shows the FEA model of the rectangular microgripper. Similar to the cantilever beam simulation here also Cosolve (coupled analysis of MemMech 


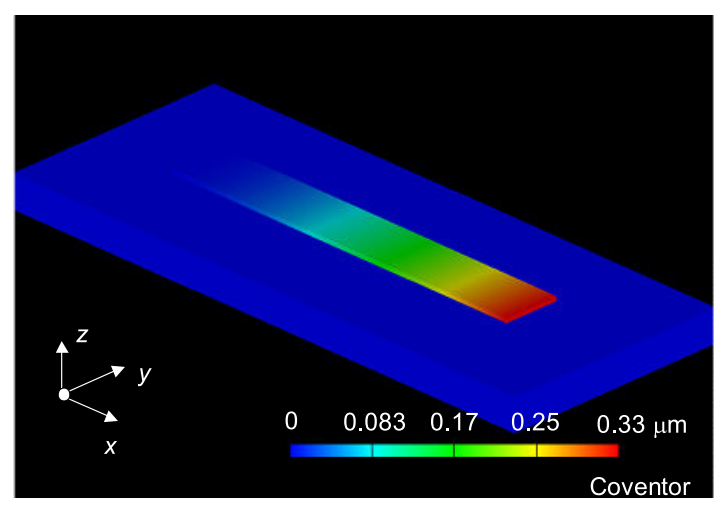

Fig. 4. FEA simulation model in CoventorWare

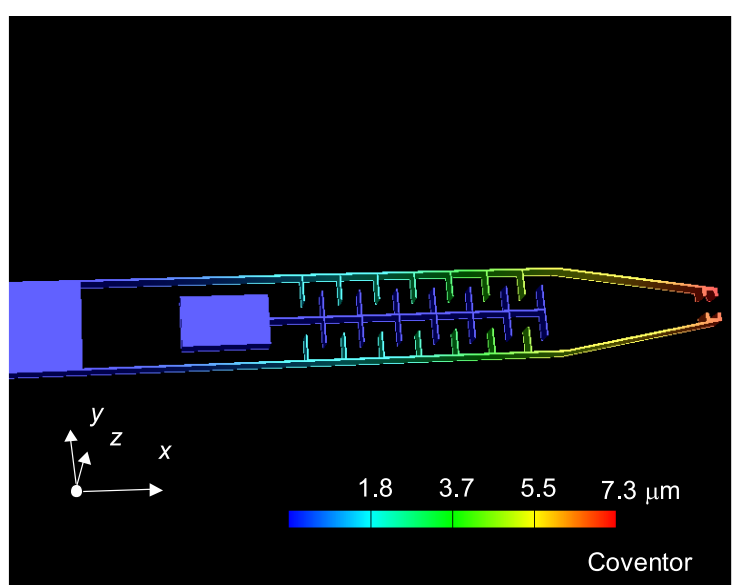

Fig. 6. FEA simulation of microgripper

and MemElectro) of CoventorWare is used to detect the pull-in voltage. The pull-in voltage comparison for rectangular microgripper is given in Tab. 5 .

With reference to Tab. 1 from the previous cantilever beam pull-in voltage results for a dimension range of $w / d_{0} \leq 2, V_{P I, 1}$ is a better suited model with less error. Here those results are further validated by microgripper. For dimension range of $w / d_{0} \leq 2, V_{P I, 1}$ shows a less error of $1.202 \%$, compared to the other models.

\section{CONCLUSION}

In this paper five closed form models for the calculation of pull-in voltages have been derived from different capacitance models. These models are validated by comparing the results with Cosolve FEA results for wide range of dimensions. Comparison of the pull-in voltages determined from these models shows that particular models are better suited for particular given ranges. The error is within $\pm 2 \%$ deviation as compared to the experimentally verified results for those particular models. It is observed that to get accurate value of pull-in voltage, appropriate models have to be chosen for the respective range of dimensions. Further these models have been validated

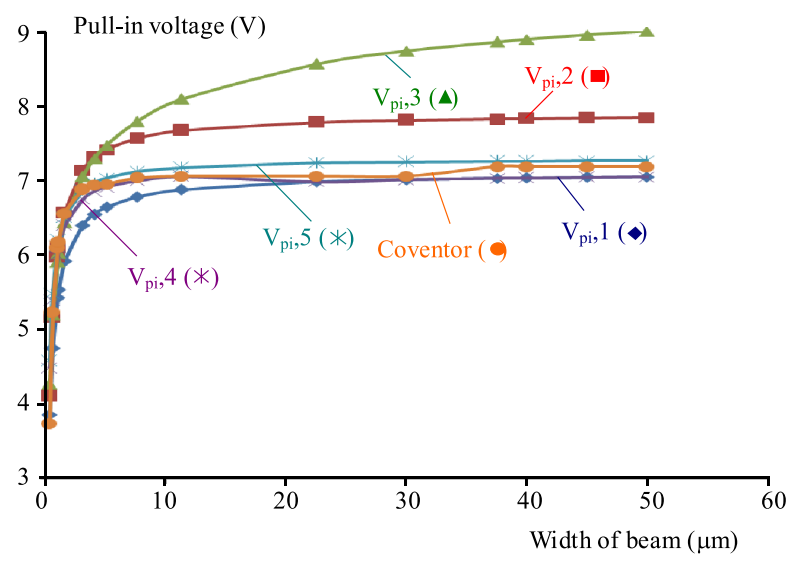

Fig. 5. Pull-in voltage versus width of the beam

by finding the pull-in voltage of electrostatically actuated microgripper with rectangular fingers. These models are relatively simple and less time consuming against the FEA models.

\section{Acknowledgement}

The authors would like to thank NPMASS program (National Program on Microstructure and Smart Systems) for their support in providing the MEMS simulation tools and UGC (University Grants Commission) of Government of India for their financial grant.

\section{Appendix - Pull-in voltage models}

All formulae bellow are presented in a uniform fashion, using reduced (dimensionless) variables

$$
r=\frac{d_{0}}{h}, \quad s=\frac{w}{h} .
$$

\section{Model 1}

$$
V_{P I, 1}=\frac{h}{l^{2}} \sqrt{\frac{0.222}{c_{f} A_{1} r} \frac{\tilde{E}}{\varepsilon_{0}}}
$$

where dimensionless function

$$
A_{1}=\frac{5}{6}+0.189\left(\frac{r}{s}\right)^{0.75}+0.398\left(\frac{r}{s^{2}}\right)^{0.5}
$$

and the compensation factor was set to: $c_{f}=0.76$.

\section{Model 2}

$$
\begin{aligned}
& V_{P I, 2}=\frac{h}{l^{2}} \sqrt{\frac{0.222}{c_{f} B_{1} r} \frac{\tilde{E}}{\varepsilon_{0}}} \\
& \text { where } B_{1}=\left[\frac{5(2 s-1)}{12 s^{2}}\right]+\pi\left(\frac{r}{s}\right)^{2} \frac{\frac{(r+1)+r}{\sqrt{r(r+1)}}+2}{\sigma \log \sigma^{2}}+ \\
& +2 \pi r\left[\frac{16 r(r+1)+\log \sigma(2 r+1) \sqrt{r(r+1)}}{3 s^{2} \log \sigma^{3}(r+1)^{2}}\right]
\end{aligned}
$$

where $\sigma=1+2 r+2 \sqrt{r(r+1)}$, and the compensation factor was set to: $c_{f}=0.76$. 


\section{Model 3}

$$
\begin{aligned}
& V_{P I, 3}=\frac{h}{l^{2}} \sqrt{\frac{0.222}{c_{f} C_{1} r} \frac{\tilde{E}}{\varepsilon_{0}}} \\
& \text { where } C_{1}=\frac{5}{6}+\frac{5 r+4}{3 r^{2}}+\frac{2 r^{2}}{3(r+1)(2 r+2+s)^{2}}+ \\
& +\frac{5}{3 s} \log \frac{2 r+2+s}{2 r+2}+\frac{r(6 r+5)}{3(r+1)^{2}(2 r+2+s)}
\end{aligned}
$$

and the compensation factor was set to: $c_{f}=0.50$.

\section{Model 4}

$V_{P I, 4}=\frac{h}{l^{2}} \sqrt{\frac{0.222}{c_{f} D_{1} r} \frac{\tilde{E}}{\varepsilon_{0}}}$

where $D_{1}=0.958+0.438 \frac{r^{0.778}}{s}$

and the compensation factor was set to: $c_{f}=0.80$.

\section{Model 5}

$$
\begin{aligned}
& V_{P I, 5}=\frac{h}{l^{2}} \sqrt{\frac{0.222}{c_{f} E_{1} r} \frac{\tilde{E}}{\varepsilon_{0}}} \\
& \text { where } E_{1}=\frac{5}{36}+\frac{4 r}{3 \pi s}
\end{aligned}
$$

and the compensation factor was set to: $c_{f}=0.89$.

\section{REFERENCES}

[1] SENTURIA, S. D.: Microsystems Design, Kluwer Academic, Boston, MA, 2000, pp. 249-259.

[2] BARKE, E. : Line to Ground Capacitance Calculation for VLSI: A Comparison, IEEE Transactions on ComputerAided Design $\mathbf{7}$ No. 2 (Feb 1988).

[3] BATRA, R. C.-PORFIRI, M.-SPINELLO, D.: Review of Modeling of Electrostatically Actuated Micromechancial System, J. Smart Mater. Struct. 16 No. 6 (Dec 2007), R23-R21, DOI: 10.1088/0964-1726/16/6/R01.

[4] CHANG, W. H. : Analytical IC Metal Line Capacitance formulas, IEEE Transactions in Microwave Theory and Techniques (Sep 1976).

[5] Van Der MEIJS, N. P.-FOKKEMA, J. T.: VLSI Circuit Reconstruction from Mask Topology, Integration 2 No. 2 (Mar 1984), 85-119.

[6] YUAN, C. P.-TRICK, T. N.: Simple Formulas for the Two and Three Dimensional Capacitances, IEEE Conf. Comput-Aided Design (1984), 263-265.

[7] ELMASRY, M. I.: Capacitance Calculations in MOSFET VLSI, IEEE Electron Device Letts. EDL-3 No. 1 (Jan 1982), 6-7, DOI: 10.1109/EDL.1982.25454.

[8] SAKURAI, T.-TAMARU, K. : Simple Formulas for Two- and Three-Dimensional Capacitances, IEEE Trans. Electron Devices ED 30 No. 2 (Feb 1983), 183-185, DOI: 10.1109/TED.1983.21093.

[9] PALMER, H. B.: The Capacitance of a Parallel-Plate by the SchwartzChristoffel Transformationjour Trans. AIEE.

[10] CHOWDhurY, S.-AhMADI, M.-MILLER, W. C.: A Closed-Form Model for the Pull-in Voltage of Electrostatically
Actuated Cantilever Beams, J. Micromech Microeng 15 No. 4 (Apr 2005), 756-763.

[11] OSTERBERG, P. M.: Electrostatically Actuated Micromechanical Test Structures for Material Property Measurements, PhD Dissertation, Massachusetts Institute of Technology, MA, 1995.

[12] LADABAUM, I.-JIN, X.-SOH, H. T.-KHURIYAKUB, B. T. : Surface Micromachined Capacitive Ultrasonic Transducers, IEEE Transactions on Ultrasonic and Ferro Electronics, Freq. control. 45 (1999), 679-90.

[13] OSTERBERG, P. M.-SENTURIA, S. D. : M Test: a Test Chip for MEMS Material Property Measurement using Electrostatically Actuated Test Structures, Journal of Micro Electro Mechanical Systems 6 No. 2 (June 1997), 107-118.

[14] TILMANS, H. A. C.-LEGTENBERG, R.: Electrostatically Driven Vacuum-Encapsulated Polysilicon Resonators Part II. Theory and Performance, Sensors and Actuators A Physical 45 No. 1 (Oct 1994 ,), 67-84, DOI: 10.1016/0924-4247(94)00813-2.

[15] NEMIROVRSKY, Y.-BOCHOSZA-DEGANI, O. : A Methodology and Model for the Pull-in Parameters of Electrostatic Actuators, Journal of Micro Electro Mechanical Systems 10 No. 4 (Dec 2001), 600-615.

[16] HU, Y. C. : Closed Form Solutions for the Pull-in Voltage of Microcurled Beam Subjected to Electrostatic Loads, J. Micromech. Microeng 16 (Feb 2006), 648-655.

[17] HU, Y. C.-LEE, G. D. : A Closed Form Solution for the Pull-in Voltage of the Micro Bridgejour Tamkang Journal of Science and Engineering.

[18] RAMEZAN, A.-ALASTY, A.-AKBARI, J.: Closed Form Solutions of the Pull-in Instability in Nano Cantilevers under Electrostatic and Intermolecular Surface Stress, International Journal of Solids and Structures 44 (July 2007), 4925-4941.

[19] CHATERJEE, S.-POHIT, G.: A Large Deflection Model for Pull-in Analysis of Electrostatically Actuated Microcantilever Beams, Journal of Sound and Vibration 322 (Jan 2009), 964-986.

[20] VARONA, J-SAENZ, E-FISCALWOODHOUSE, S.-HAMOUI, A. A.: Design and Fabrication of a Novel Microgripper Based on Electrostatic Actuation, Circuits and Systems, 2009. MWSCAS '09. 52nd IEEE International Midwest Symposium. Aug. 2009.

Received 23 August 2011

Kalaiarasi Arcot Ramakrishnan received the AMIE degree in Electrical Engineering from the Institution of Engineer(s) India, India, and the Post graduate Degree in Control and Instrumentation from College of Engineering, Anna University, India. She is currently working toward the Ph. D degree at College of Engineering, Anna University. She is a Senior Research Fellow (SRF) of UGC (University Grants Commission), of Government of India. Her research interest is in Design and Modeling of MEMS devices, Fabrication and characterization of MEMS devices, Linear and Non-Linear Systems theory, control systems and Electrical machines

Hosimin Thilagar Srinivasan was born in 1971. He completed his BE and ME degrees from Madurai Kamaraj University in 1992 and 1994 respectively. He completed PhD at IIT Madras in 2004. He is presently working at Anna University, Chennai as Associate Professor in Department of Electrical and Electronics Engineering. His areas of interest are Electrical Machines, Drives and MEMS technology. 